$\begin{array}{rr}\text { Universitas } & \text { Vol. } 37 \\ \text { Núm. } 78\end{array}$

\title{
Nuevo aparato ortopédico fijo para el tratamiento de maloclusiones clase II división 1. Descripción de un caso*
}

\author{
New Orthopedic Fixed Appliance to Treat Class II, Division 1 Malocclusions. Case Report
}

\author{
Rosmery Cecilia García Ramos ${ }^{a}$ \\ Práctica privada, Colombia \\ orto.ros@hotmail.com \\ ORCID: http://orcid.org/0000-0003-0905-5975
}

DOI: https://doi.org/10.11144/Javeriana.uo37-78.naof Redalyc: http://www.redalyc.org/articulo.oa? id $=231260072009$

Fecha de recepción: 20 Septiembre 2017 Fecha de aprobación: 07 Junio 2018 Fecha de publicación: 18 Junio 2018

\section{Resumen:}

Antecedentes: La corrección temprana de las maloclusiones clase II asociadas a retrognatismo mandibular se logra mediante un avance mandibular sostenido y continuo que no requiera colaboración del paciente. Objetivo: Describir un caso de maloclusión tratado con un nuevo arco de adelantamiento mandibular fijo (AAMF). Descripción del caso: El AAMF se usó en una niña de 11 años y 6 meses de edad, clase II esquelética división 1, con retrognatismo mandibular y mordida profunda. Resultados: $\mathrm{Al}$ año de tratamiento había cambios estéticos y funcionales. El ángulo ANB disminuyó $\left(6,6^{\circ}\right.$ a $\left.5,0^{\circ}\right)$. La longitud efectiva mandibular (Co-Gn) aumentó $(96,1 \mathrm{~mm}$ a $103,4 \mathrm{~mm})$. La inclinación de incisivos superiores pasó de $28,0^{\circ}$ a $28,4^{\circ}$. Los incisivos inferiores se proinclinaron levemente $\left(26,2^{\circ}\right.$ a $\left.30,9^{\circ}\right)$. Las cefalometrías después de la fase de ortodoncia mostraron medidas esqueléticas estables: ANB pasó de $5,0^{\circ}$ a $5,1^{\circ}$ y Co-Gn se mantuvo en $103,4^{\circ}$. Hubo cambios en la posición de los incisivos: superiores retroinclinaron de $28,4^{\circ}$ a $22,5^{\circ}$ e incisivos inferiores pasaron de $30,9^{\circ}$ a $28,2^{\circ}$. Conclusión: El AAMF representa una alternativa de tratamiento en denticiones mixta y permanente temprana: Por ser fijo, permite lograr avance mandibular sostenido y continuo, sin requerir colaboración del paciente, y durante el tiempo necesario para generar cambios. Por la sencillez de su estructura (maxilar cementado solo en los primeros molares maxilares), avanza la mandíbula sin fijarse a ella cada vez que el paciente cierra la boca. Además, es sencillo de elaborar, cementar, activar, limpiar y mantener en boca.

Palabras clave: aparatos ortopédicos fijos, avance mandibular, maloclusiones, maloclusiones clase II, odontología, ortodoncia, ortopedia, ortopedia maxilar, retrognatismo mandibular, tratamiento de maloclusiones clase II con aparatología fija.

\begin{abstract}
:
Background: Early correction of class II malocclusions associated to mandibular retrognathism is possible through sustained and continuous mandibular advancement that does not require patient collaboration. Purpose: To describe a case of malocclusion treated with a new fixed mandibular advance arch (FMAA). Case description: FMAA was applied to a 11-year-6-monthold skeletal class-II, division-1 girl with mandibular retrognathism and overbite. Results: After one-year treatment, there were functional and esthetic outcomes. ANB angle was reduced from $6.6^{\circ}$ to 5.0 $0^{\circ}$. Effective mandibular length (Co-Gn) increased ( 96.1 $\mathrm{mm}$ to $103.4 \mathrm{~mm})$. Upper incisors inclinations went from $28,0^{\circ}$ to $28,4^{\circ}$. Lower incisors experienced slight proinclinations $\left(26.2^{\circ}\right.$ to $30.9^{\circ}$ ). Cephalograms after orthodontic treatment showed stable measures: ANB went from $5.0^{\circ}$ to $5.1^{\circ}$ and Co-Gn remained stable in $103.4^{\circ}$. Upper incisors had retroinclinations from $28.4^{\circ}$ to $22.5^{\circ}$ and lower incisors went from $30.9^{\circ}$ to $28.2^{\circ}$. Conclusion: FMAA is a treatment alternative for mixed and early permanent dentitions for being a fixed appliance, it allows advancing mandible without requiring patient collaboration and during the necessary time to obtain changes. Because of its simple structure (cemented in the maxilla only to first molars), it advances without being fixed to the mandible every time the patient closes the mouth. In addition, it is easy to make, cement, activate, clean, and keep in mouth.
\end{abstract}

Keywords: class II malocclusions, class II malocclusion treatment with fixed appliances, dentistry, malocclusions, mandibular advance, mandibular retrognathism, maxillary orthopedics, orthodontics, orthopedic fixed appliances, orthopedics.

Notas de autor: 


\section{Introducción}

Una de las maloclusiones más frecuentes y que causa mayor desarmonía facial, capaz de generar un impacto psicológico negativo, es la clase II división 1, caracterizada por retrognatismo mandibular, mordida con resalte (overjet) aumentada, clase molar y canina II, mordida profunda, perfil convexo y tercio inferior disminuido. Estas condiciones exigen que los músculos faciales y de la lengua se adapten a patrones anormales de contracción. Típicamente, el músculo mentoniano es hiperactivo, el labio superior es hipotónico y el inferior es hipertónico. En casos severos, los incisivos superiores descansan sobre el labio inferior (1). En la etiología de estas maloclusiones se definen dos componentes principales que interactúan: predisposición genética y factores exógenos o ambientales que se presenten durante el desarrollo craneofacial, $(2,3,4)$ o durante la niñez, como hábitos orales nocivos, que dependiendo de la frecuencia, duración e intensidad pueden crear cambios específicos en la oclusión (5).

Harris y Kowalski (3) comprobaron mediante estudios cefalométricos. el potencial hereditario de la maloclusión clase II división 1. Junto a esta influencia genética, muchos trabajos destacan la importancia del medio ambiente en la etiopatogenia de las distoclusiones. Por ejemplo, la hipótesis de la matriz funcional, postulada por Melvin Moss (6,7), sugiere que el cartílago nasal y todo el conjunto de suturas que rodean el maxilar inferior son centros de crecimiento compensatorio sujetos a control ambiental. Las maloclusiones clase II división 1 pueden deberse a una displasia ósea básica por discrepancia sagital en el crecimiento de los maxilares. Este crecimiento se lleva a cabo a través de la aposición ósea en los cóndilos mandibulares y en el circuito sutural que une el complejo nasomaxilar con el cráneo, siguiendo una trayectoria hacia adelante y abajo. Sus superficies externas son remodeladas hasta alcanzar el tamaño, la morfología y la posición topográfica de la cara adulta. También puede producirse una clase II división 1 por el movimiento de los arcos dentarios, el superior hacia adelante y el inferior hacia atrás, debido a hábitos como la succión digital y la succión del labio inferior A través del tiempo, los enfoques para tratar las maloclusiones clase II división 1 se han fundamentado en a) restringir el crecimiento maxilar y estimular o redirigir el crecimiento mandibular mediante el remodelado condilar y de la fosa glenoidea por uso simultáneo y combinado de cráneo-maxilares y aparatos funcionales $(8,9,10)$, y b) estimular o redirigir el crecimiento mandibular $(9,10,11)$, mediante aparatos funcionales removibles y fijos $(12,13,14,15,16,17)$.

No existe un patrón de referencia (gold standard) específico entre estos aparatos para el tratamiento de las maloclusiones clase II división 1; la selección la determina solo el criterio del clínico, y entre los de mayor evidencia en la literatura están el twin-block $(18,19)$ y el Herbst $(20,21)$. Los inconvenientes de la aparatología funcional removible y fija existente son: el gran volumen intraoral bimaxilar, lo cual los hace incómodos, y el uso a intervalos muy cortos, que depende de la colaboración del paciente para producir cambios significativos en el tiempo necesario. En el campo de la ortopedia maxilar se ha evidenciado la necesidad de encontrar aparatología funcional fija que supere las inconvenientes de la aparatología ya existente. No existe en la literatura sobre el tema ningún aparato funcional similar al usado en este caso clínico y su uso representa una alternativa que supera los inconvenientes de la aparatología ya existente.

\section{Materiales y método}

Los datos del presente informe se obtuvieron de una niña de 11 años y 6 meses de edad con diagnóstico de maloclusión clase esquelética II división 1 con retrognatismo mandibular y mordida profunda. El tratamiento se aprobó y la madre de la paciente firmó el consentimiento informado. La fotometría extraoral mostró un biotipo mesofacial, simetría facial, perfil convexo asociado al retrognatismo mandibular y labio inferior evertido (figura 1). 


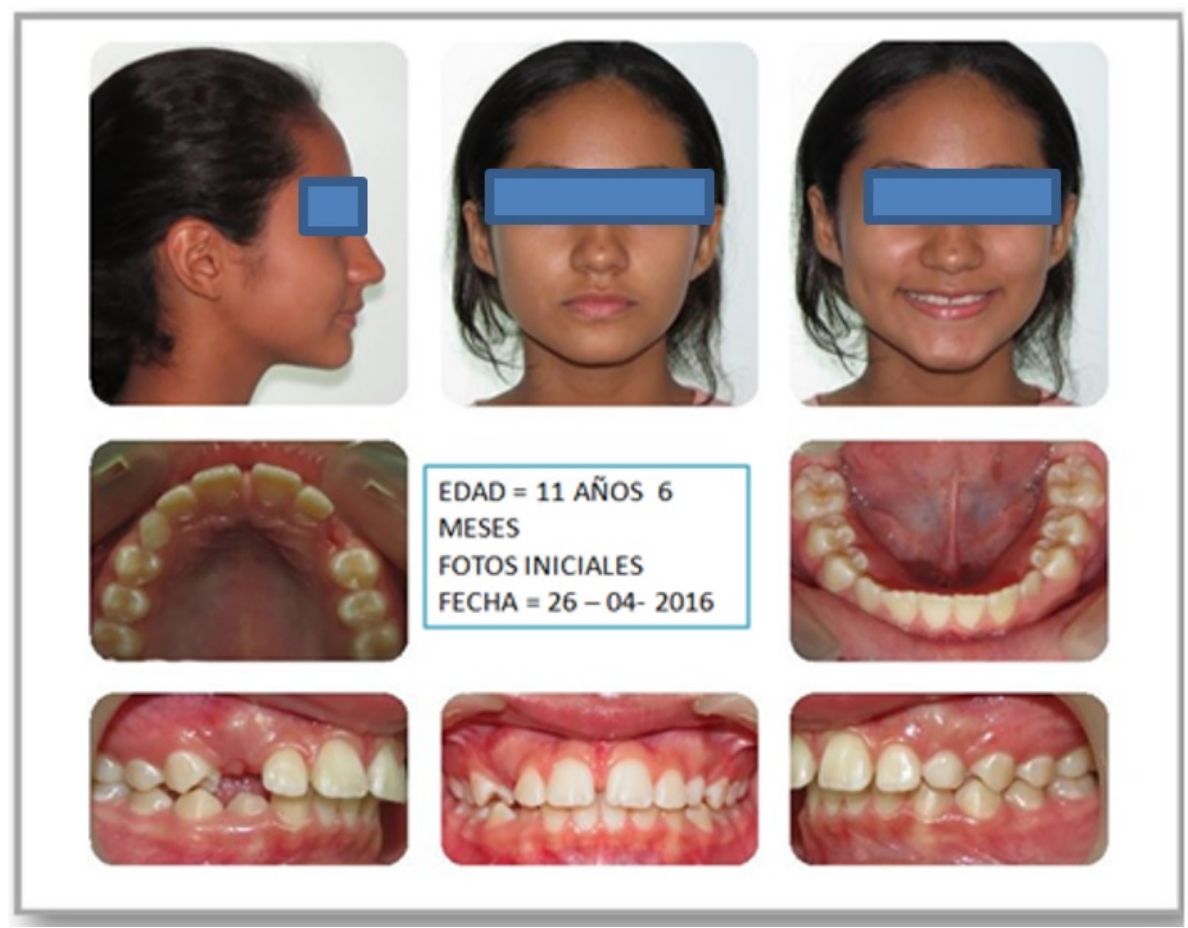

FIGURA 1

Fotografías extraorales e intraorales de la paciente

Fuente: elaboración propia.

La fotometría intraoral, la radiografía panorámica y los modelos de estudio mostraron que la paciente estaba en la etapa de dentición mixta, con incisivos, premolares y primeros molares superiores inferiores erupcionados; caninos inferiores en erupción, 13 y 23 incluidos. La línea media superior e inferior eran coincidentes. Sobremordida de $5 \mathrm{~mm}$ con incisivos inferiores tocando el paladar; curva de Spee invertida; mordida de resalte de $7 \mathrm{~mm}$, sin apiñamiento y relación molar clase II. La radiografía de perfil reveló la discrepancia en la relación maxilo-mandibular. $\mathrm{El}$ análisis de maduración de las vértebras cervicales, según Baccetti, muestra un estadio CS3 donde los bordes de C4 están planos, C2 y C3 presentan concavidad, y C3 y C4 tienen forma trapezoidal, lo que indica que el pico de crecimiento está en proceso y es el momento ideal para realizar el avance mandibular ortopédico. El análisis cefalométrico de Steiner (figura 2) mostró una clase II esquelética, con ángulo $\mathrm{ANB}=6,6^{\circ}$, retrognatismo mandibular con ángulo $\mathrm{SNB}=82,4^{\circ}$, inclinación de Is $(\mathrm{U} 1-\mathrm{NA}=28,0)$, inclinación de $\mathrm{Ii}\left(\mathrm{Li}-\mathrm{NB}=26,2^{\circ}\right)$. La longitud mandibular inicial ( $\left.\mathrm{Co}-\mathrm{Gn}\right)$ fue de 96,1 $\mathrm{mm}$, según el análisis cefalométrico de McNamara (12) (figura 3). 


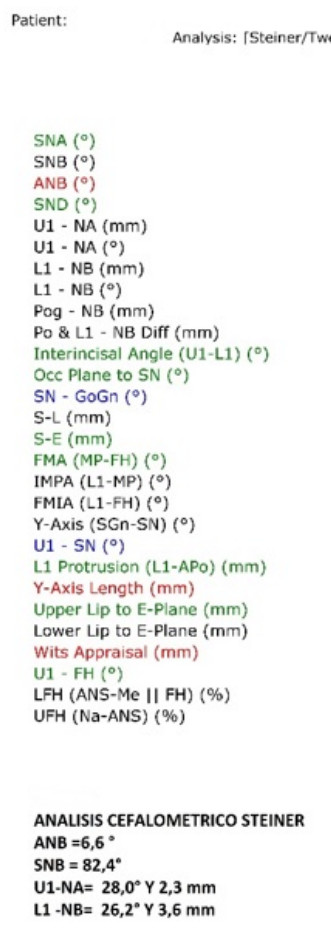

$\mathrm{L} 1-\mathrm{NB}=26,2^{\circ} \mathrm{Y} 3,6 \mathrm{~mm}$

ID: 1103739290, Female, Age: $13 y$ 9m (DOB: 25/10/2004)
d/Wits) Norm: N/A

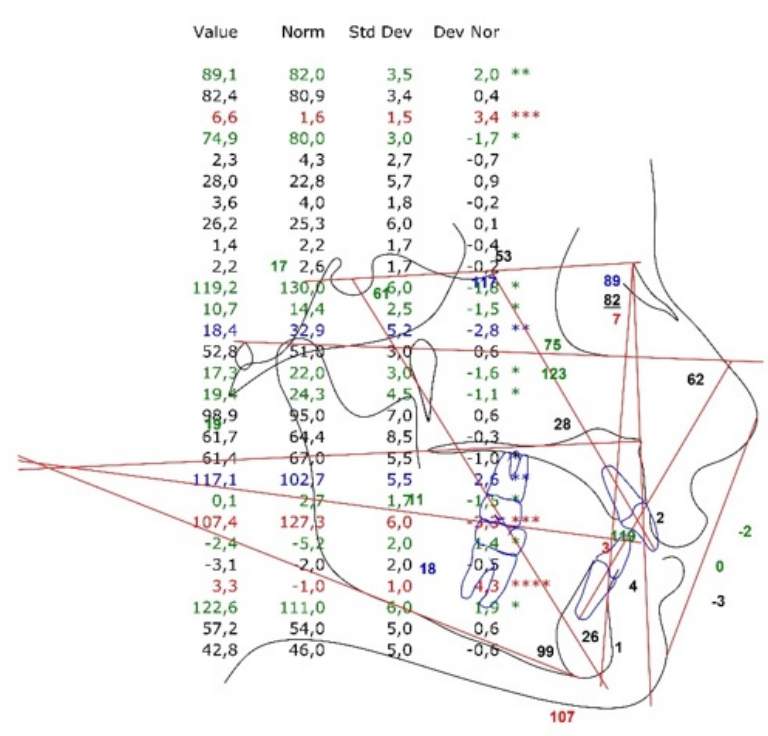

FIGURA 2

Análisis cefalométrico de Steiner

Fuente: elaboración propia.

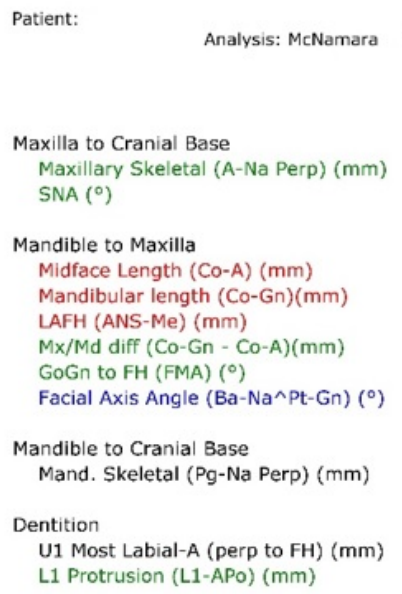

ID: 1103739290 , Female, Age: $13 y$ 9m (DOB: 25/10/2004)

Maxilla to Cranial Base

Maxillary Skeletal (A-Na Perp) (mm)

Norm: N/A

L1 Protrusion (L1-APo) (mm)

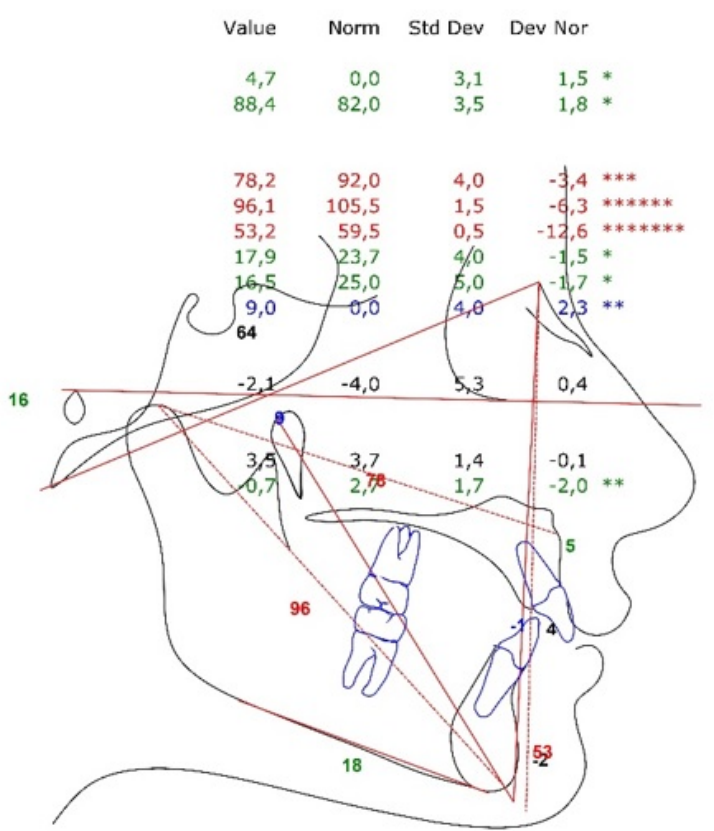

FIGURA 3

Análisis cefalométrico de McNamara

Fuente: elaboración propia. 
Para fabricar el arco de adelantamiento mandibular fijo (AAMF), la autora del aparato que aquí se presenta tomó una impresión del maxilar superior de la paciente, con alginato (Tropicalgin Zhermack), previa adaptación de bandas prefabricadas de acero en los primeros molares maxilares. Sobre el modelo de trabajo obtenido se fabricó un arco de Acero 0,9 y se extendió anteriormente sobre el paladar sin tocarlo hasta llegar frente de la cara palatina de los centrales superiores permanentes, haciendo una $U$ hacia el plano oclusal. Este arco se soldó a la cara palatina de las bandas prefabricadas ubicadas en los primeros molares maxilares. Si miramos el aparato lateralmente, esta $U$ forma un ángulo más o menos agudo dependiendo de la mordida constructiva que requiera la maloclusión de cada paciente y actúa como un tope que avanza la mandíbula cada vez que el paciente cierra la boca (figura 4).

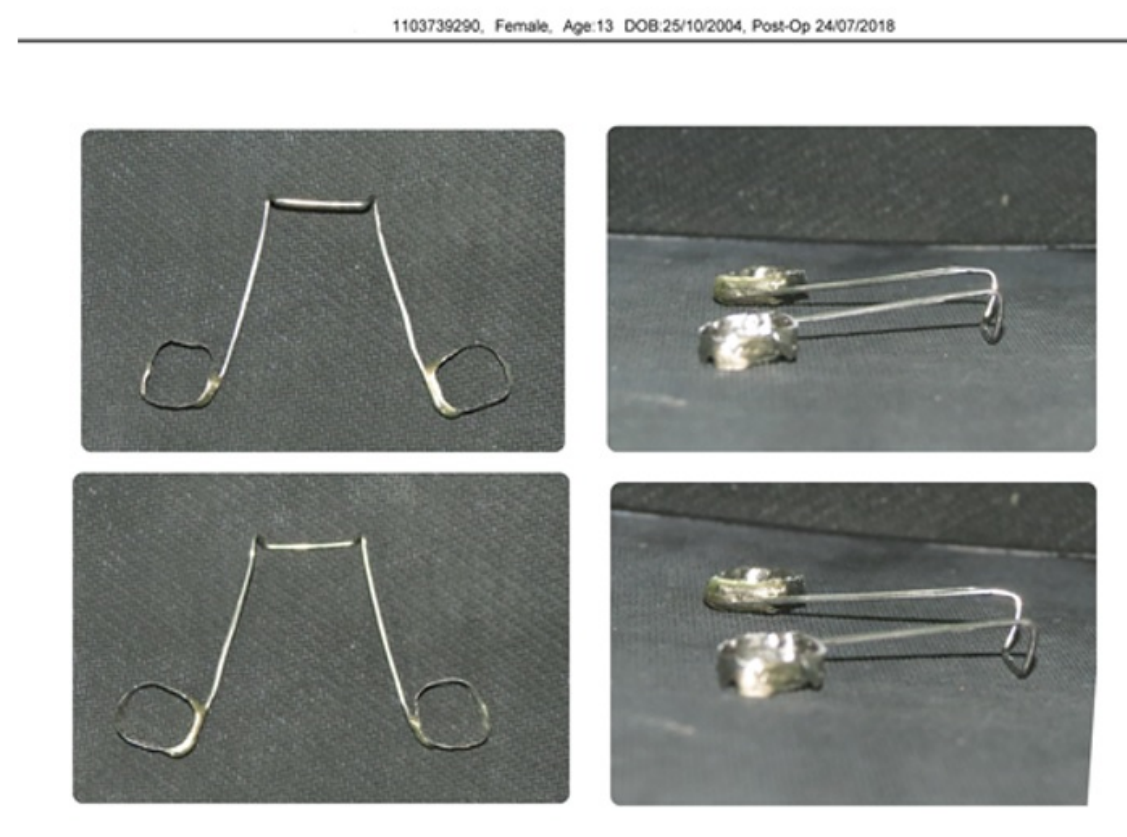

APARATO

FIGURA 4

Arco de adelantamiento mandibular fijo

Fuente: elaboración propia.

Una vez cementado el aparato con ionómero de vidrio (Ideal plus USA), se observa acople solo entre los incisivos superiores y los inferiores, quedando en inoclusión el área de caninos y premolares. Entre las características diferenciadoras con respecto a otros aparatos funcionales fijos, podemos mencionar que la fuerza de tracción anterior aplicada a los músculos pterigoideos laterales es continua y constante, que no requiere la colaboración del paciente, lo que ocasionará una respuesta bioquímica en la región del cartílago condilar, coronoides y angular de la mandíbula que hace posible modular la magnitud del crecimiento en esas áreas (13). Debido a su diseño sencillo y unimaxilar, aporta comodidad al clínico para su elaboración, cementación, activación, y al paciente para su uso, mantenimiento e higiene.

\section{Presentación del caso}

Durante la fase de tratamiento se hicieron controles mensuales para verificar la higiene y el estado del aparato en cuanto a función y posición. En una ocasión hubo que bajar el aparato y volver a soldarlo, pues se fracturó la unión entre el arco y la banda del 16. A los 6 meses se observó clínicamente un perfil más recto con aumento del tercio inferior en altura y una proyección anterior del mentón. Después de un año de tratamiento, tiempo 
mínimo previsto según el análisis de Baccetti para observar pico de crecimiento máximo en el paciente y cambios significativos en el crecimiento mandibular, en la fotometría extraoral postratamiento se detalló que mejoraron el perfil de tejidos blandos, la relación labial y el tercio inferior (figura 5).
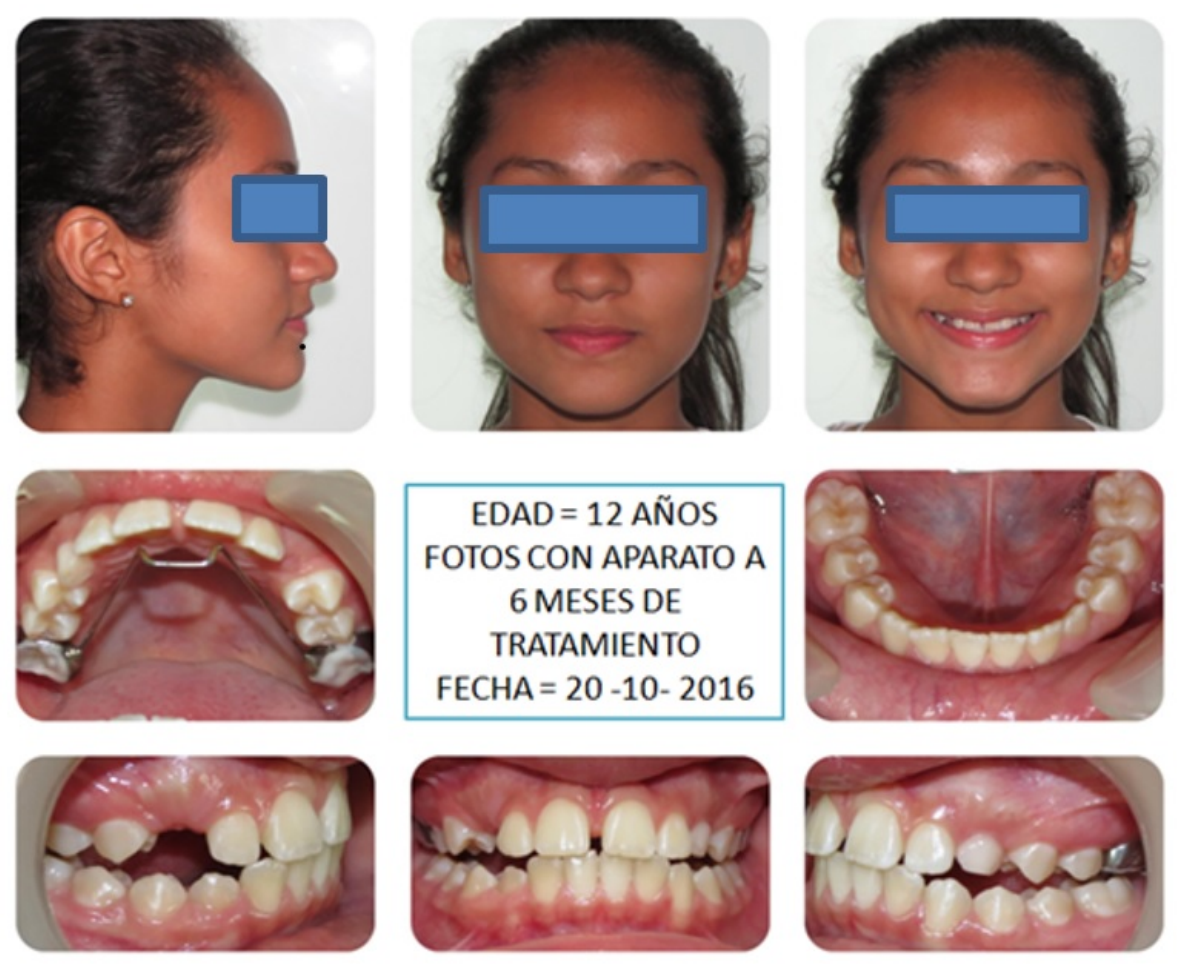

FIGURA 5

Fotografías de la paciente un año después

Fuente: elaboración propia.

Clínicamente, y en los modelos, se observó que la relación molar pasó de clase II a clase I; los caninos permanentes erupcionaron levemente rotados, en clase I. La curva de Spee se aplanó con una buena intercuspidación de los premolares. En la radiografía de perfil se observó mejoría en la relación maxilomandibular, mordida con resalte y sobremordida. La radiografía panorámica mostró buena relación ósea y periodontal; el tercio inferior de los premolares en formación; segundos molares en proceso de erupción y germen de los terceros molares. La cefalometría de Steiner mostró un descenso del ángulo ANB, que pasó de 6,6 a 5, $0^{\circ}$ (figura 6). En la cefalometría de McNamara, la longitud mandibular (Co-Gn) aumentó, al pasar de $96,1 \mathrm{~mm}$ a $103,4 \mathrm{~mm}$ (figura 7 ). 


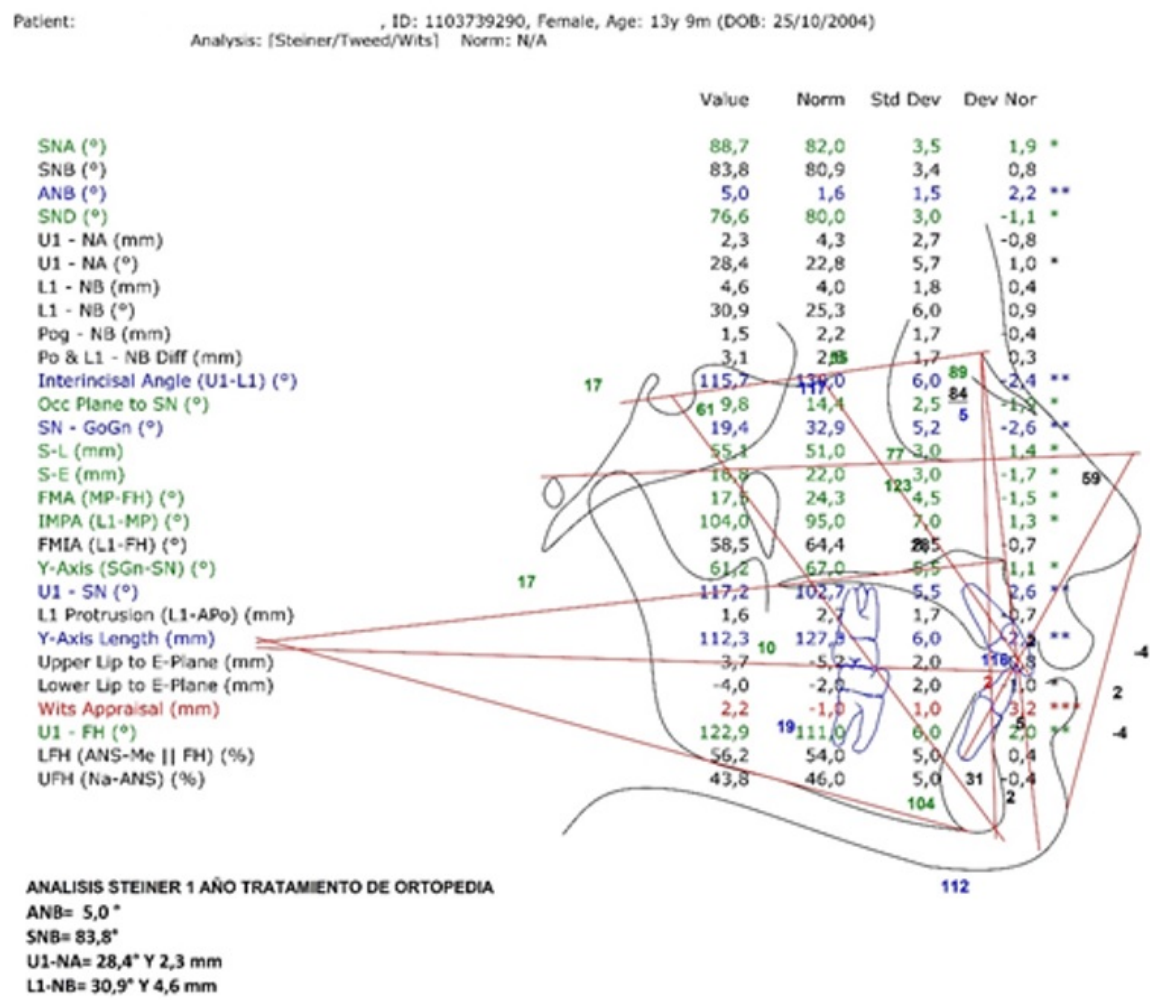

FIGURA 6

Cefalometría de Steiner después de un año Fuente: elaboración propia.

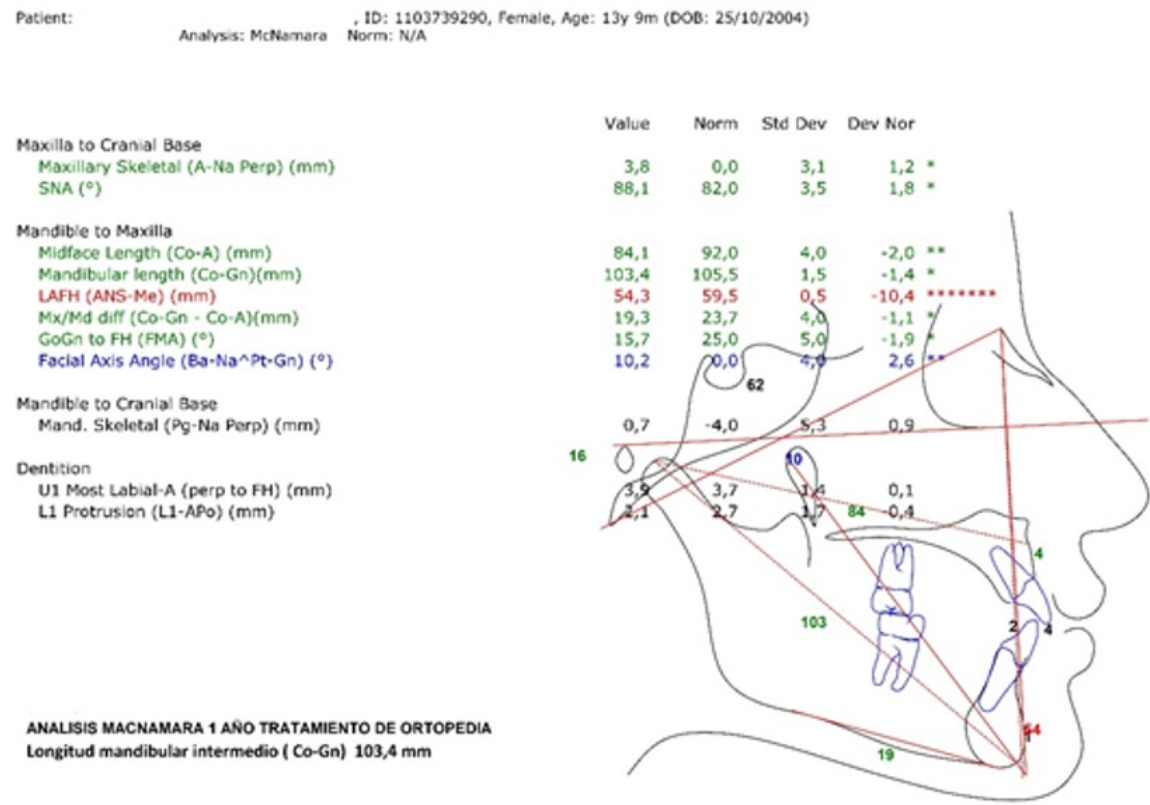

FIGURA 7

Cefalometría de McNamara después de un año

Fuente: elaboración propia.

La inclinación de los incisivos superiores pasó de $28,0^{\circ}$ a $28,4^{\circ}$. Los incisivos inferiores se proinclinaron levemente: de $26,2^{\circ}$ a $30,9^{\circ}$. Completado un año con el aparato AAMF, se retiró y se inició la fase de ortodoncia correctiva. Un año posterior al tratamiento de ortopedia y después de la fase de ortodoncia se 
realizó gingivoplastia del sector anterosuperior para lograr mejor estética y se observó que las relaciones caninas y molares estaban en clase I bilateral con buen acople anterior y overjet-overbite. Se le tomó a la paciente una radiografía de perfil donde se realizaron las cefalometrías de Steiner y McNamara para valorar si hubo recidiva de la corrección ortopédica. El análisis de Steiner mostró un ángulo ANB de $5,1^{\circ}$ y la inclinación de los incisivos superiores (U1-NA: 22, $5^{\circ}$ ) y los incisivos inferiores (Li-NB: 28, $2^{\circ}$ ) (figura 8). El análisis de McNamara mostró una longitud mandibular (Co-Gn) de 103,4 mm (figura 9).

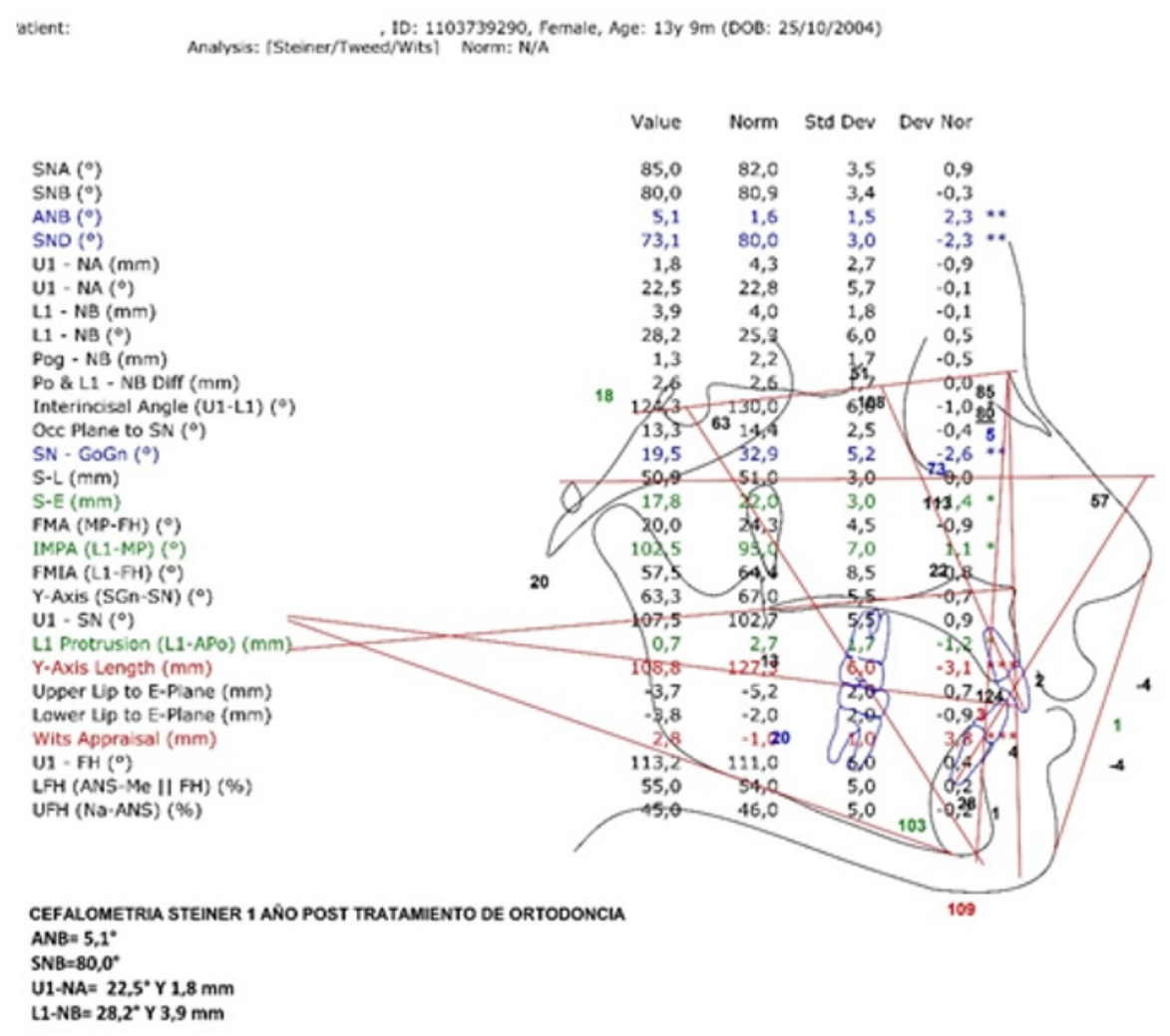

FIGURA 8

Análisis de Steiner sobre recidiva de la corrección ortopédica

Fuente: elaboración propia. 


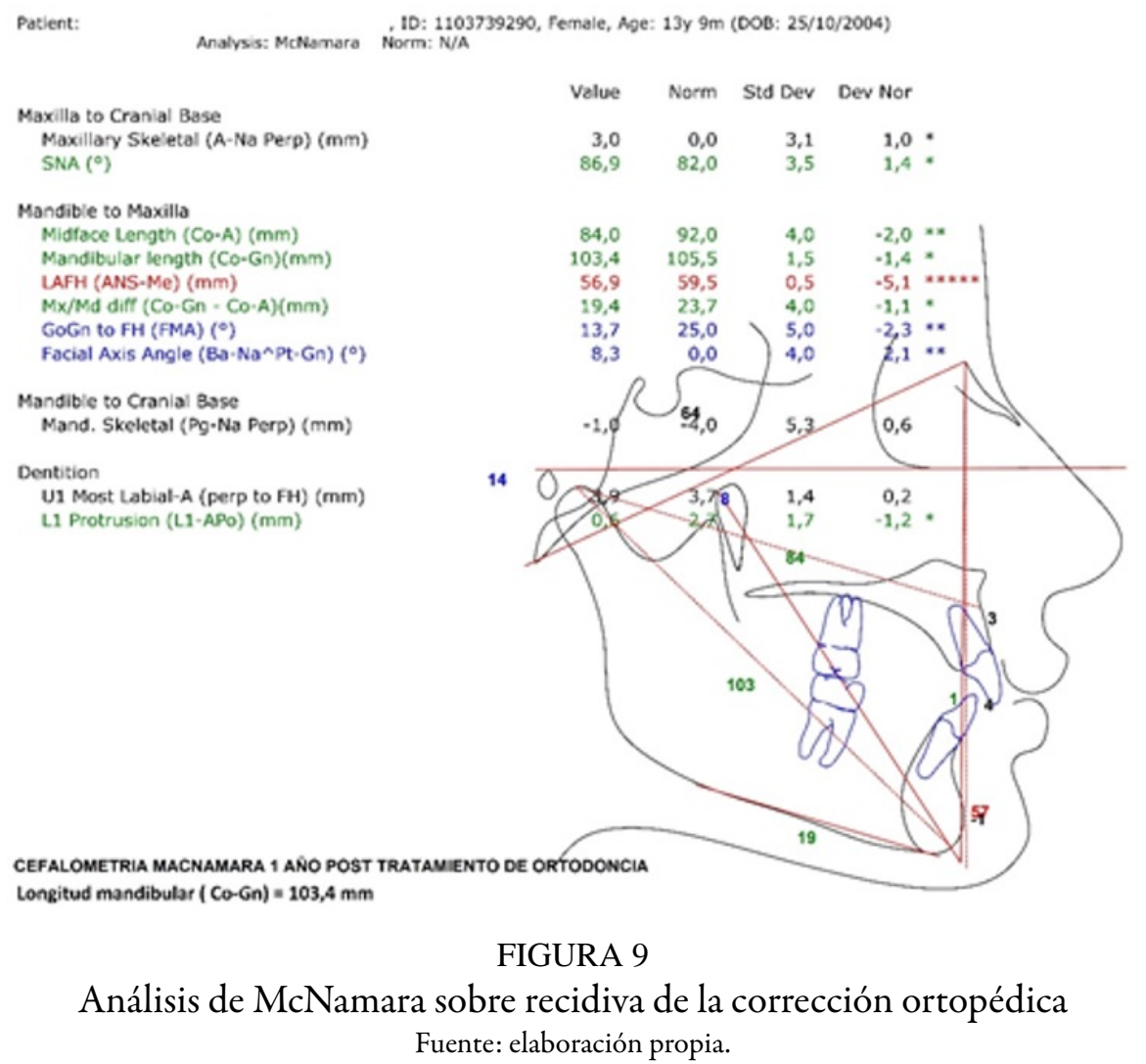

Esto sugiere que los cambios ortopédicos esqueléticos se mantuvieron en un periodo considerable posterior al tratamiento de ortopedia, pero que la posición de incisivos superiores e inferiores se modificó debido al tratamiento de ortodoncia posterior (figura 10). 

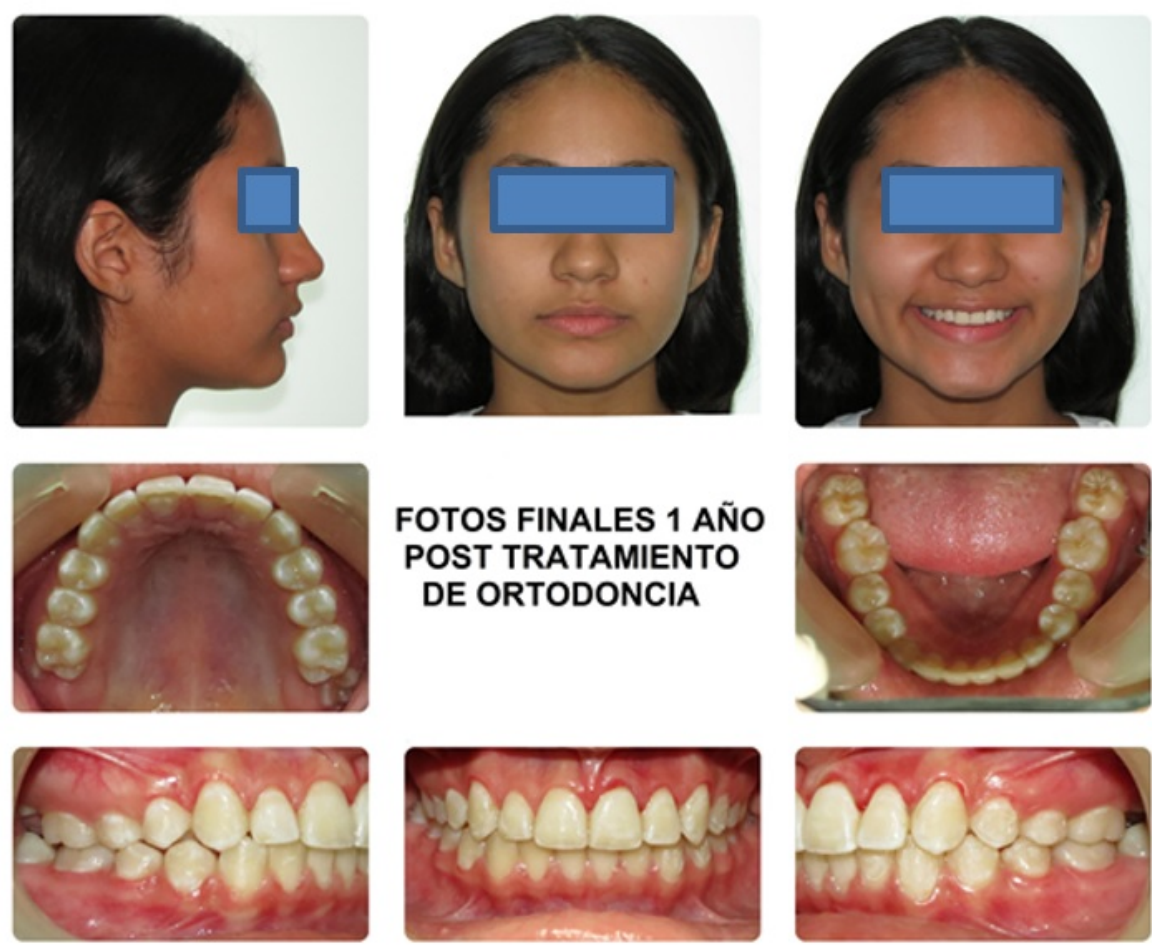

FIGURA 10

Resultados del tratamiento de ortodoncia

Fuente: elaboración propia.

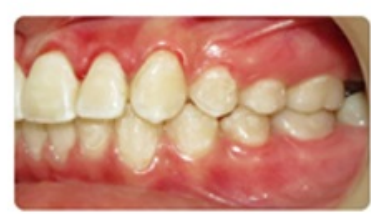

\section{Discusión}

Considerando el diagnóstico de la paciente: clase II esquelética división 1 con micrognatismo mandibular anteroposterior (ángulo ANB: 6,6 $6^{\circ}$ y que según el análisis de maduración de las vértebras cervicales de Baccetti estaba en el momento ideal para avance mandibular ortopédico, se decidió aprovechar esto para corregir la discrepancia orientando el crecimiento mandibular usando el nuevo aparato AAMF. La importancia de la interacción entre el músculo pterigoideo lateral y la almohadilla retrodiscal en el crecimiento mandibular ha sido demostrada en diferentes investigaciones en ratas $(14,15)$. El uso de un dispositivo de adelantamiento mandibular provoca una actividad contráctil en el músculo pterigoideo lateral que se traduce en un doble efecto mecánico y circulatorio sobre el cartílago condilar, lo que podría regular su desarrollo, corrigiendo las discrepancias maxilo-mandibulares originadas por micrognatismo mandibular anteroposterior.

La corrección de la discrepancia sagital maxilomandibular durante el pico máximo de crecimiento es fundamental para lograr un equilibrio neuromuscular del sistema estomatognático, de modo que los dientes puedan ubicarse de acuerdo con este, hasta lograr una buena oclusión, ya sea naturalmente o usando aparatología ortodóncica adicionalmente, en una etapa posterior.

Esta paciente hubiera podido tratarse con otros aparatos ortopédicos funcionales removibles, como el Twin Block $(18,19)$, o fijos, como el Herbst $(16,20,21)$ y el Jasper Jumper (22), que reportan efectos similares al del AAMF, pero considerando sus desventajas como el ser removibles o fijos (bimaxilares, voluminosos e incómodos), generalmente, los pacientes no los usan durante el tiempo requerido para generar cambios sustanciales, por lo cual se decidió usar el nuevo aparato AAMF, que supera estos inconvenientes por ser fijo, sencillo en su estructura unimaxilar y cómodo en el uso e higiene. 
Entre los hallazgos esqueléticos favorables encontrados en las cefalometrías, cabe resaltar que hubo un incremento en la longitud mandibular (Co-Gn), que pasó de $96,1 \mathrm{~mm}$ a 103,4 $\mathrm{mm}$ (figura 7), y disminución del ángulo $\mathrm{ANB}$, que pasó de $6,6^{\circ}$ a 5,0 (figura 6), y que esta corrección se ha mantenido un año después del tratamiento de ortopedia $\left(\mathrm{ANB}=5,1^{\circ}\right)$ y Co-Gn $(103,4 \mathrm{~mm})$ (figuras 8 y 9 ), contrario a otros estudios con Herbs, con resultados similares, pero a corto plazo (21). Hubo un hallazgo posiblemente desfavorable: la leve proinclinación que sufrieron los incisivos inferiores, que pasaron de $26,2^{\circ}$ a $30,9^{\circ}$ (figura 6 ), mencionado también en otros aparatos funcionales fijos como el Herbst (21) y el Jasper Jumper (22). Respecto al tiempo indicado para iniciar el tratamiento de ortopedia, la literatura informa que los cambios esqueléticos favorables son menores cuando se inicia el tratamiento mucho antes del pico de crecimiento (23), por lo cual se determinó iniciar en la etapa CS3 de Baccetti.

Franco y colaboradores (17) mostraron en un estudio con resonancia magnética que el desplazamiento del disco articular no es una complicación de la terapia con aparatos funcionales en pacientes clase II división 1 tratados durante la pubertad. No se encontraron hallazgos de dolor o alteraciones articulares y musculares durante el uso del aparato. Entre las posibles desventajas que podrían presentarse con el AAMF están la fractura de la soldadura o descementación del aparato, como ocurrió durante este tratamiento. Al introducir este nuevo prototipo del aparato AAMF se abre la puerta para realizar futuros estudios clínicos controlados aleatorizados, con un diseño mejorado y validar la actividad de la musculatura comprometida en el proceso. El AAMF ha sido usado en otros casos clínicos todavía no reportados.

\section{Conclusiones}

De acuerdo con los resultados del caso clínico descrito, se concluye que con el uso del nuevo aparato AAMF hubo cambios esqueléticos evidenciados así. El ángulo ANB pasó de $6,6^{\circ}$ a 5, $0^{\circ}$ y la longitud mandibular CoGn pasó de $96,1 \mathrm{~mm}$ a 103,4 mm. También hubo cambios dentales como la relación molar, que pasó de clase II a clase I; los incisivos superiores, de $28,0^{\circ}$ a $28,4^{\circ}$; y los incisivos inferiores se proinclinaron levemente, pasando de $26,2^{\circ}$ a $30,9^{\circ}$. Los cambios esqueléticos se mantuvieron estables un año después de terminada la corrección ortopédica (ANB: $5,1^{\circ}$ ), así como la longitud mandibular (Co-Gn: 103,4 mm), por lo cual se recomienda usar el AAMF como una alternativa de tratamiento para las maloclusiones clase II división 1 a temprana edad y realizar nuevos ensayos clínicos controlados aleatorizados para comprobar hallazgos cuantificables.

\section{Agradecimientos}

La autora agradece al doctor Luis Rogelio Hernández, por el procesamiento de los datos del estudio.

\section{Referencias}

1. Cassidy SE, Stona R, Jackson D, Turpin L, Ramsay DS, Spiekerman C, Hueng GJ. Classification and treatment of class II subdivision malocclusions. Am J Orthod Dentofacial Orthop. 2014; 145(4): 443-51. https://doi.org/1 0.1016/j.ajodo.2013.12.017

2. Harris EF, Johnson MG. Heritability of craniometric and occlusal variables: a longitudinal sib analysis. Am J Orthod Dentofacial Orthop. 1991 Mar; 99(3): 258-68. https://doi.org/10.1016/0889-5406(91)70007-J

3. Harris, JE, Kowalski CJ. All in the family: use of familial information in orthodontic diagnosis, case assessment and treatment planning. Am J Orthod. 1976; 69(5): 493-510. https://doi.org/10.1016/S0002-9416(76)80022-X

4. Mendoza Oropeza L, Melendez Ocampo O, Ortiz Sanchez R, Fernández López A. Prevalence of malocclusions associated with pernicious oral habits in a Mexican sample. Rev Mex Ortod. 2014; 2(4): 216-23. https://doi.o rg/10.1016/S2395-9215(16)30151-9 
5. King L, Harris EF, Tolley EA. Heritability of cephalometric and occlusal variables as assessed from siblings with overt malocclusions. Am J Orthod Dentofacial Orthop. 1993 Aug; 104(2): 121-31. https://doi.org/10.1016/ S0889-5406(05)81001-7

6. Moss ML. The functional matrix hypothesis revisited. 3. The genomic thesis. Am J Orthod Dentofacial Orthop. 1997 Sep; 112(3): 338-42. https://doi.org/10.1016/S0889-5406(97)70265-8

7. Moss ML. The functional matrix hypothesis revisited. 4. The epigenetic antithesis and the resolving synthesis. Am J Orthod Dentofacial Orthop. 1997 Oct; 112(4): 410-7. https://doi.org/10.1016/S0889-5406(97)70049-0

8. Yokota S, Murakami T, Shimizuk A. A grow control approach to class 2 division 1, cases during puberty involving the simultaneous application of maxillary grow restriction and mandibular forward. Am J Orthod Dentofacial Orthop. 1993; 104 (3): 211-23. https://doi.org/10.1016/S0889-5406(05)81722-6

9. Keerthi VN, Kanya SD, Babu K, Mathew A, Kumar AN. Early prevention and intervention of class II division 1 in growing patients. J Int Soc Prev Community Dent [internet]. 2016; 6(suppl 1): 579 -83. Disponible en: http:/ /www.jispcd.org/text.asp?2016/6/7/79/181191

10. Marsan G. Effects of activator and high-pull headgear combination therapy: skeletal, dentoalveolar and soft tissue profile change. Eur J Orthod. 2007; 29(2): 140-8. https://doi.org/10.1093/ejo/cjm003

11. Hagg U. Change in mandibular growth direction by means of Herbst appliance. A case report. Am J Orthod Dentofacial Orthop.1992; 102(5): 456-63. https://doi.org/10.1016/S0889-5406(05)81193-X

12. McNamara JA Jr, Peterson JE Jr, Alexander RG. Three-dimensional diagnosis and management of class 2 malocclusion in the mixed dentition. Semin Orthod. 1996; 2(2): 114-37.

13. Rabie AB, She TT, Harley VR. Forward mandibular positioning up-regulates SOX9 and type II collagen expression in the glenoid fossa. J Dent Res. 2003 Sep; 82(9): 725-30.

14. Satoh K. Mechanical advantage of area of origin for the external pterygoid muscle in two murid rodents, Apodemus speciosus and Clethrionomys rofocanus. J Morphol. 1999; 240(1): 1-14. https://doi.org/10.1002/ (SICI)1097-4687(199904)240:1<1::AID-JMOR1 >3.0.CO;2-D

15. Zhou ZL, Huel Xi, Qiang Y, XueZa Z Differential expression of 16F-1 and its mRNA in mandibular condylar cartilage of rat. Direct evidence for servosystem theory of facial growth. Hua Xi Kou Qiang Yi Xue Za Zhi. 1998 May; 16(2):164-5.

16. Graber T, Rakosi T, Petrovic A. Ortopedia con aparatos funcionales. 2a ed. New York: Harcourt Brace; 1998.

17. Franco AA, Yamashita HK, Lederman HM, Cavidanes LH, Proffit WR, Vigorito JW. Frankel appliance therapy and the temporomandibular disc: a prospective magnetic resonance imaging study. Am J Orthod Dentofacial Orthop. 2002; 121(5): 447-57. https://doi.org/10.1067/mod.2002.122241

18. Toth L, McNamara JA Jr. Treatment effects produced by the twin- block appliance and the FR-2 appliance of Frankel compared with an untreated class II sample. Am J Orthod Dentofacial Orthop. 1999 Dec; 116(6): 597-609.

19. Parra Quintero N, Botero Mariaca MP. Aparatos de avance mandibular: ¿ Mito o realidad? Rev Nac Odontol. 2012 dic; 9(edición especial): 57-73.

20. Canut BL. Ortodoncia clínica y terapéutica. $2^{\mathrm{a}}$ ed. Barcelona, España: Masson; 2000.

21. Pancherz H The Herbst appliance: Its biologic effects and clinical use. Am J Orthod. 1985; 87(1): 1-20.

22. Herrera FS, Henrriques JF, Janson G, Franciscon MF, de Freitas KM. Cephalometric evaluation in different phases of Jasper Jumper therapy. Am J Orthod Dentofacial Orthop. 2011 Aug; 140(2): e77-84. https://doi.org/10.10 16/j.ajodo.2011.03.018

23. De Almeida MR, Henriques JF, de Almeida RR, Weber U, McNamara JA Jr. Short-term treatment effects produced by the Herbst appliance in the mixed dentition. Angle Orthod. 2005; 75(4): 540-7. http://dx.doi.org/10.1590 /S1415-54192006000500004 


\section{Notas}

* Artículo de investigación.

Licencia Creative Commons CC BY 4.0

Cómo citar este artículo: García Ramos RC. Nuevo aparato ortopédico fijo para el tratamiento de maloclusiones clase II división 1. Descripción de un caso. Univ Odontol. 2018 ene-jun; 37(78). https://do i.org/10.11144/Javeriana.uo37-78.naof 